\title{
CAEP/ACMU 2017 Scientific Abstracts Keyword Index
}

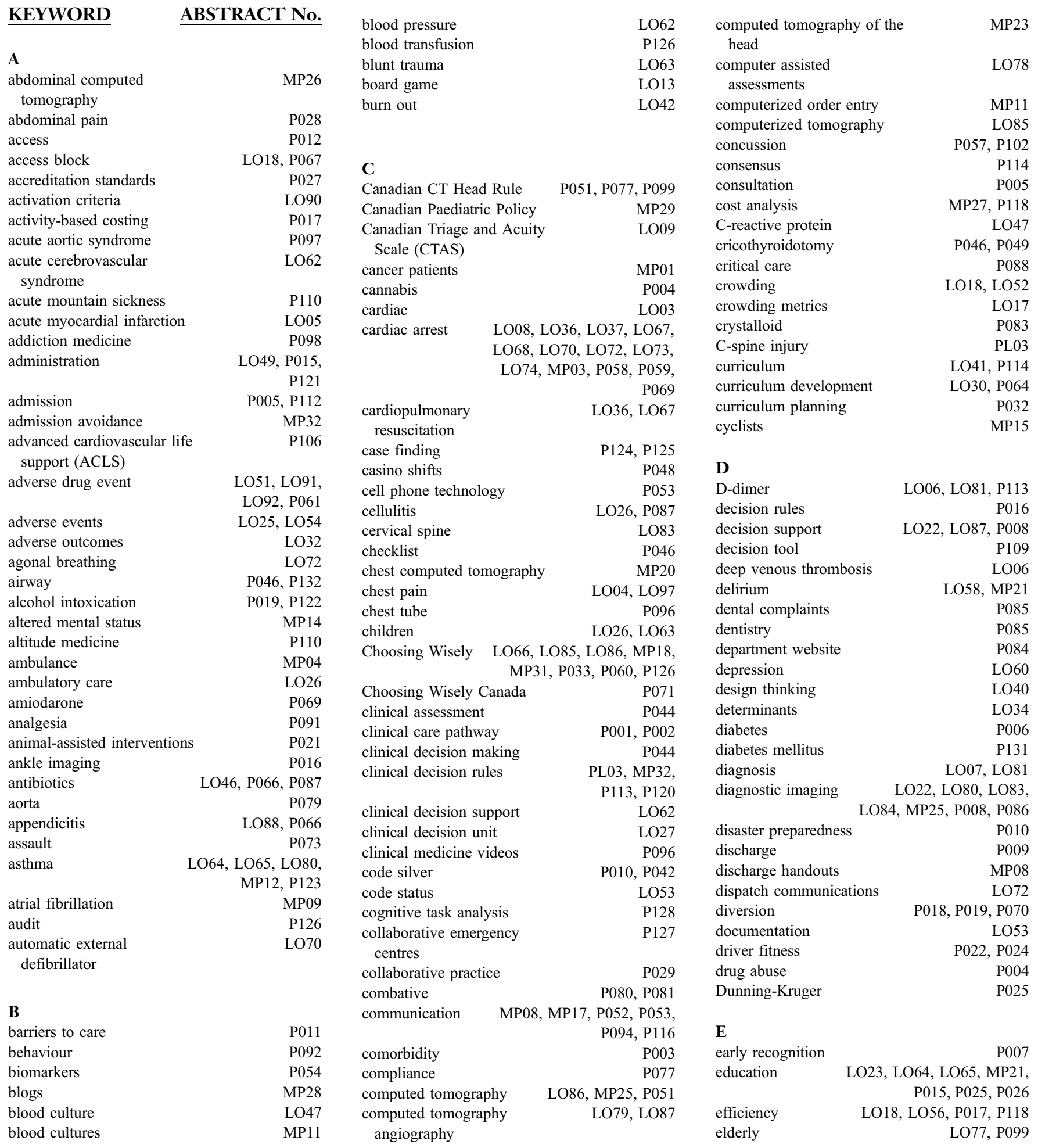


electrocardiograph
monitoring

LO98

electrolytes

emergency

PL02,

emergency department

P076

P16, P012, P085, P093

LO02, LO12, LO50
LO52, LO66, LO80,

MP05, MP12, MP26,

MP33, P021, P070,

P071, P072, P076, P094, P119, P124, P125

emergency department care

emergency department cost

emergency department

crowding

emergency department

discharge

emergency department flow

emergency department systems

transformation (EDST)

emergency medical services

4, LO31

LO35, LO59, P055,

LO67, P080,

P081, PL03

emergency medical services

MP36

bypass

emergency medical services

operations

emergency medicine

LO43, LO44, LO45, LO78, P041, P108, P117

emergency medicine

education

emergency physician

end of life care

entrustment

epidemiology

equity

erysipelas

ethics

exercise

exercise prescription

expertise

extracorporeal

cardiopulmonary

resuscitation

extracorporeal resuscitation

eye-tracking

\section{F}

faculty development

falls

fast track

fentanyl

first trimester

fluid bolus

fracture

frailty

free open access medical education (FOAM)

LO50, LO61, MP12, P040

P047

P014

$\mathrm{P} 075$

P040
P121

P087

P038

P095

P092

P128

LO68

frequent users

G

geriatric PL02, LO73, LO76, LO77, MP13,

P039

P128

$\mathrm{P} 104$
$\mathrm{MP} 07$

MP10

LO76, P002

PL02, LO78, P054

LO14, LO28,

LO30, MP35,

$\mathrm{P} 032$

LO49 gestalt

global health education

global health partnership

global health research

global health training

goals

guidelines

guns

\section{H}

handover

head injury

headache

health care utilization

health policy

health promotion

health services

health system design

health systems

heart failure

hip fracture

history of emergency

medicine

history of medicine

human immunodeficiency

virus (HIV)

human trafficking

hyperemesis

hypoglycemia

hypotension

\section{I}

imaging

implementation

in situ simulation

incidental findings

infographics

injection

injury

injury prevention

innovation

innovations in emergency medicine education

input mitigation

interactive group tool

inter-professional education

interrater agreement

intervention

intimate partner violence

intubation

$\mathbf{K}$

knowledge translation LO22, MP31, P008, P033, P084, P120

$\mathbf{L}$

laboratory medicine

laboratory testing

lactate

leadership

length of stay

levels of evidence

$\mathrm{P} 074$

LO15

P063

LO15

LO15

$\mathrm{P} 013$

LO24

P040

MP17

LO66, MP18, P122

LO50, LO84, MP25

P119

P121

P092

LO91

P029

LO55

PL01, LO02

LO58

P065

P065

P073

P045

$\mathrm{P} 004$

LO38, P006

LO07, LO43, LO44, LO45

MP06

P009, P071

$\mathrm{P} 010$

MP20

MP22

MP06

$\mathrm{P} 054$

MP29, P040

MP35

P045, P100, LO20,

P130, LO14, LO28,

LO29, LO42, P064,

$\mathrm{P} 065$

LO16

LO19

P041

LO75

LO83, P086

MP05, P124, P125

MP34, P132

MP14, P009, P115

low back pain

lumbosacral imaging

P086

P060

M

machine learning LO40, P03

mass gathering $\quad$ P129

massive online needs LO30

assessment

medical education

MP28, P045,

P096, P098, P102

medication reconciliation LO51

mental health LO59, MP33, P013, P018,

P062, P111, P119

mentorship

meta-analysis

LO21

metatarsal

midazolam

mild traumatic brain injury

(mTBI)

minor head injury

minor injury

minor stroke

mobility

morbid obesity

morphine

mortality

motor vehicle crashes

multisource feedback

myocardial infarction

LO93

P002

P080, P081

LO95, P057,

P077, P078

P051, P099

P115

LO61

P115

P058

LO05

LO05, LO63, P056

$\mathrm{P} 022, \mathrm{P} 024$

$\mathrm{P} 030$

LO04, LO97

\section{$\mathbf{N}$}

naloxone

P105

near-peer teaching

LO20

needs assessment $\quad \mathrm{P} 032$

nephrolithiasis LO82

neural network

$\mathrm{P} 031$

night shifts

nitroglycerin

non-operative management

LO48, P048

LO33

P066

P078

non-return to work

non-traumatic low back pain

P060

P020

nurse education

P027

\section{O}

occupational injury

LO95

offload

MP04

offload delay

online medical education

LO32, LO34

LO29

LO41

opioid

, P104

optimization

P067

MP09

P075

oral case presentation

MP06

osteoarthritis

MP14 


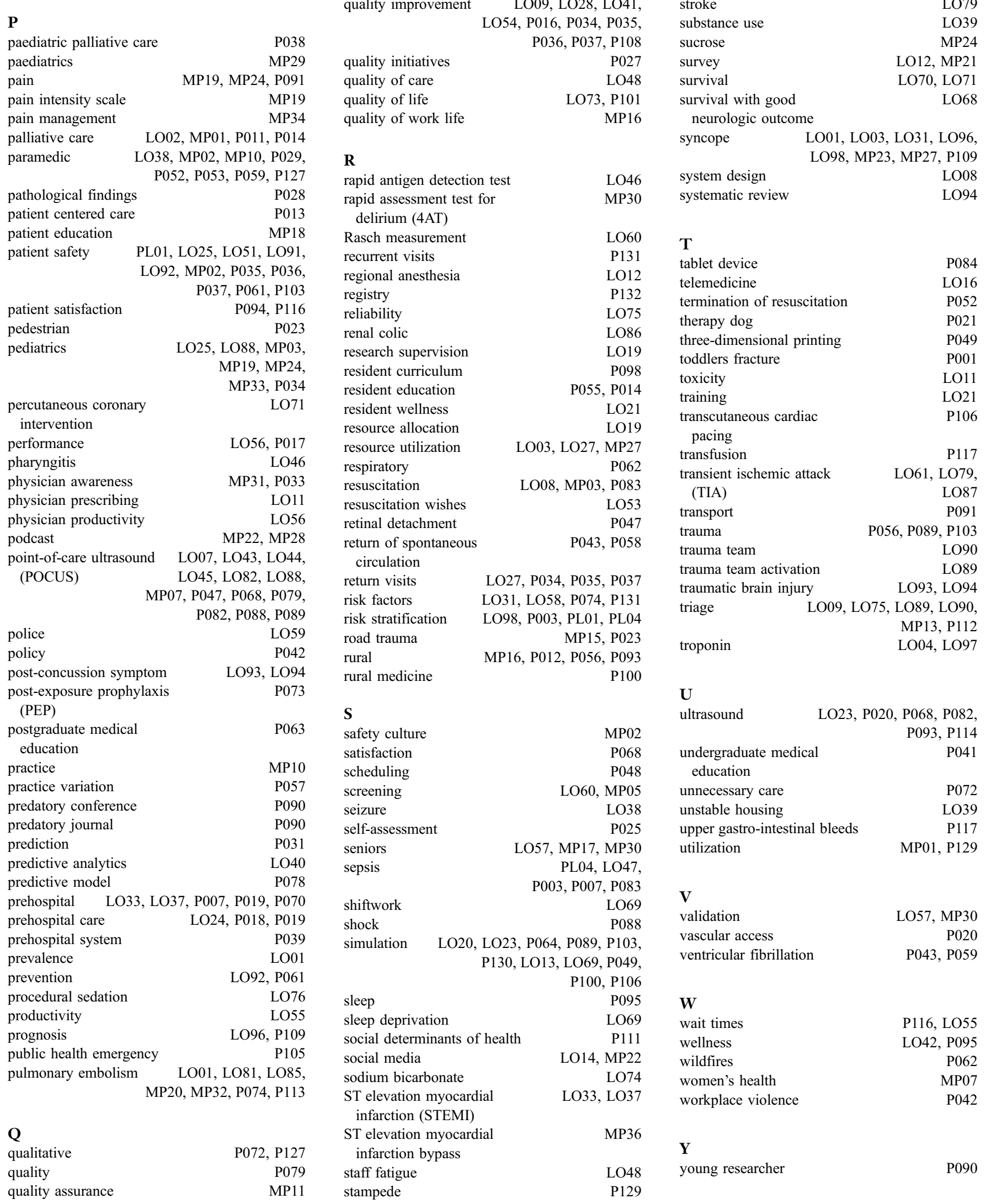

